\title{
Portulaca oleracea L. Polysaccharides enhances immune efficacy of Dendritic Cells Vaccine in Breast Cancer
}

\author{
Rui Zhao ${ }^{1}$, Guiyan Jia ${ }^{1}$, Xingyue Shao ${ }^{2}$, Tao Zhang ${ }^{3}$, Xiechen Zhou ${ }^{1}$, Yang Yang ${ }^{1}$, Tao Li $^{1}$, \\ Zhao Chen ${ }^{1}$, Yupeng Liu ${ }^{1}$, and Jinzuo Hou ${ }^{1}$ \\ ${ }^{1}$ Heilongjiang August First Land Reclamation University \\ ${ }^{2}$ Daqing Oilfield General Hospital \\ ${ }^{3}$ Jiamusi University
}

September 10, 2020

\begin{abstract}
Background and purpose Previous studies have reported that Portulaca oleracea L. polysaccharides (POL-P3b) is an immunoregulatory agent. However, POL-P3b as a novel immune adjuvant in combination with DC vaccine for breast cancer treatment has been little studied. In this work, DC vaccine loaded with mouse 4T1 tumor cell antigen was prepared to evaluate the properties of POL-P3b in inducing maturation and function of DC derived from mouse bone marrow, and then to investigate the effect of DC vaccine combined with POL-P3b on breast cancer in vivo and in vitro. Experimental approach Morphological changes of DC were observed using scanning electron microscopy. Phenotypic and functional analysis of DC were detected by flow cytometry and allogeneic lymphocyte reaction. Cytokine levels in DC culture supernatant were detected by ELISA. Western blotting analysis for the protein expression of TLR4, MyD88 and NF- $\varkappa$ B. Apoptosis detection and protein expression of tumor tissue were analysed by TUNEL staining and immunohistochemistry, respectively. To evaluate the security of POL-P3b by detecting hematological, blood biochemical indicators and pathological analysis for liver and kidney Key results POL-P3b can induce DC activition and maturation, attributing to increasing specific anti-tumor immune response, and the mechanism of action involved in the TLR4/MyD88/NF- $\varkappa \mathrm{B}$ signaling pathway. Experimental results in vivo further suggested that administration of POL-P3b-treated antigen-primed DC achieved remarkable tumor growth inhibition through inducing apoptosis. Moreover, POL-P3b-treated DC vaccine was able to inhibit lung metastases. Conclusions and implications The results proved the feasibility of POL-P3b as an adjuvant of DC vaccine for anti-breast cancer therapy.
\end{abstract}

\section{Hosted file}

manuscript.doc available at https://authorea.com/users/357857/articles/480237-portulacaoleracea-1-polysaccharides-enhances-immune-efficacy-of-dendritic-cells-vaccine-inbreast-cancer

\section{Hosted file}

Figure.docx available at https://authorea.com/users/357857/articles/480237-portulacaoleracea-1-polysaccharides-enhances-immune-efficacy-of-dendritic-cells-vaccine-inbreast-cancer

\section{Hosted file}

Table.doc available at https://authorea.com/users/357857/articles/480237-portulaca-oleracea1-polysaccharides-enhances-immune-efficacy-of-dendritic-cells-vaccine-in-breast-cancer 